\title{
A Comprehensive Review of Characterization and Detection of Adulteration of Extra Virgin Olive Oil
}

\author{
Rania I.M. Almoselhy*
}

Oils and Fats Research Department, Food Technology Research Institute, Agricultural Research Center, Giza, Egypt

\begin{abstract}
Determination of authenticity of extra virgin olive oils has become very important in recent years due to the increasing public concerns about possible adulterations with relatively cheap vegetable oils and deodorized olive oils. The analysis and authentication of extra virgin olive oil (EVOO) represent very challenging analytical chemical problems. Each country may have its own definition of olive oil and its own limits for particular oil parameters and monitoring guidelines in International Standards. Generally, these standards are based on the International Olive Council (IOC) and Codex Standards but often there are alternatives for different countries. The aim of this review is to provide specific information about olive oils with special concern about extra virgin olive oil (EVOO) and analytical techniques to detect adulteration of EVOO. The guidelines for genuine olive oil are adapted from the IOC, other legitimate and appropriate sources. The contents provide information on possible adulterants in olive oil, the underlying causes of adulteration, and how to test for the presence of these adulterants.
\end{abstract}

Keywords: Extra Virgin Olive Oil; Detection of Adulteration; International Standards

\section{Introduction}

Olive is the fruit of the olive tree (Olea europaea, Oleaceae). The fruit is an oval-shaped drupe composed of a pericarp (skin and flesh) and the endocarp (seed or pit). The seed contributes $15-30 \%$ of the weight of the olive depending on the cultivar. The olive contains around $50 \%$ water and $20 \%$ oil, although these proportions vary widely among olive cultivars and the environmental conditions in which they are grown. There are many cultivars grown worldwide and although around 600 can be found in the literature, the precise number is unknown. IOC has published a World catalogue of Olive Varieties describing 139 cultivars grown across 23 countries, which IOC claims represent almost $85 \%$ of the total world's acreage of olives [1].

Olive oil: Olive oil is the oil fraction of the olive fruit, i.e., pulp and seed. It is composed basically of triacylglycerols (triglycerides) with the predominant fatty acid being oleic acid $(55-83 \%)$. The other major fatty acids include palmitic (7.5-20\%), stearic (0.5-5\%) and linoleic (3.5-21\%). The oil contains some minor compounds including phytosterols, aliphatic alcohols, pigments, and a range of more polar phenolic compounds including hydroxytyrosol and tyrosol. Refined olive oils have considerably reduced levels of these minor compounds [1].

Pomace: Pomace is the solid waste material left after olive oil and water have been extracted by pressing or centrifugation of olives. Generally, the pomace contains $5-10 \%$ of the oil after centrifugation and may be further extracted using solvents [1].

A detailed description of the various olive oil grades is provided as following [1]:

\section{Virgin (or natural) olive oil}

The initial division is based on extraction methods used and oil is classified as "virgin" olive oil when it is extracted from the fruit by mechanical means, in a way which will not alter the oil characteristics. This process excludes oils obtained using solvents or re-esterification processes and of any mixture with oils of any other kind or source. Modern methods of extraction of virgin oil involve washing, grinding or crushing the fruit, mixing or malaxing the paste, and then centrifuging to separate the oil from the pomace. This category, virgin olive oil, can be further divided, based on oil quality, into the following four groups:

1. Extra virgin olive oil (EVOO) must meet chemical and organoleptic limits. In particular, the free fatty acids (measured as oleic acid) must be less than, or equal to $0.8 \% \mathrm{w} / \mathrm{w}$. In addition, the sensory requirements of this grade require that the oil have zero "defects" and positive attributes of fruitiness in excess of zero, based on sensory assessment.

2. Virgin olive oil must have less than, or equal to $2.0 \% \mathrm{w} / \mathrm{w}$ free fatty acids. The sensory requirements are that the oil be between 0 to 3.5 defects, as described by the IOC and greater than zero for fruitiness. 
3. Ordinary virgin olive oil is a category used by IOC but not all countries use this grade. It describes virgin olive oil with a free fatty acid level of not more than $3.3 \% \mathrm{w} / \mathrm{w}$.

4. Lampante virgin olive oil, also referred as "crude" in some standards, refers to any virgin olive oil exceeding the free fatty acid limits discussed above or has a peroxide value (a measure of vegetable oil rancidity) in excess of 20 millequivalents (mEq) peroxide oxygen per kg/oil per the IOC standard, while California's standard lowers this value to 15 . This oil is considered unfit for human consumption in this state and requires refining or is otherwise used for industrial purposes. All grades of edible virgin olive oil should have a peroxide value of less than, or equal to $20 \mathrm{mEq}$ peroxide oxygen per $\mathrm{kg} / \mathrm{oil}$. For lampante oil, there is no limit for peroxide value.

\section{Refined olive oil}

- Refined olive oil, sometimes called 'Pure Olive Oil', is olive oil suitable for consumption after it goes through a food grade refining process. Any process structure of the oil. Due to refining, the free fatty acids content is low and for this grade must be no more than $0.3 \%$ by weight.

- Olive oil composed of refined olive oil and virgin olive oil, is a blend of refined olive oil and virgin olive oil (other than lampante oil). In this case the free fatty acid must be not more than $1.0 \%$ by weight.

\section{Olive pomace oil}

- Crude olive pomace oil is the oil extracted, using solvents such as hexane, from the solid waste, after producing virgin olive oil. The oil requires refining if used for human consumption. Otherwise it is used for industrial purposes.

- Refined olive pomace oil is obtained by refining crude olive pomace oil in a way that does not change the glyceridic structure of the oil. The free fatty acid concentration must not exceed $0.3 \%$ by weight.

- Olive pomace oil composed of refined olive pomace oil and virgin olive oils. As the name implies, this grade of oil is a blend of virgin olive oil and refined olive pomace oil. The free fatty acid content should not exceed $1.0 \%$ by weight.

\section{Olive Oil Consumption Set to Outpace Production for a Change}

Worldwide olive oil production will be somewhat lower than consumption in the 2020 season. With current estimates suggesting production of around 3.11 million tons of olive oil and 3.14 million tons expected to be consumed, there could finally be some good news for producers, according to the international consultant Juan Vilar. "We expect that during this campaign, consumption will be higher than production" Vilar said at a recent Andalusian webinar about olive production, according to the Spanish news agency COPE. "In addition, price increases have been seen in all categories and this shows that although a change in strategy is necessary, it is a ball of oxygen for the traditional olive grove, which accounts for 70 percent of the harvest and that, without a doubt, has lived through some difficult times." There is also a growing role around the world for the "modern olive grove," said Vilar, which now accounts for " 40 percent of all olive oil produced" - numbers that reveal "a trending change and a reality which is constantly growing." Vilar also noted how the growing popularity of olive farming in five continents "brought to a total of 11.5 million hectares dedicated to olive trees." Those numbers pushed worldwide production to exceed 3 million tons "feeding households in more than 180 countries." [2].

\section{Adulteration of food}

Adulteration of food worldwide is a major issue for health and safety. Numerous cases of food poisoning and health issues have been reported through mislabeling of food or blend [1].

\section{Sources of information supporting confirmation of adulteration}

The adulteration of olive oil is not a new phenomenon; texts describing the substitution of olive oil with oils from poppy (Papaver somniferum, Papaveraceae) seeds, peanuts, sesame (Sesamum indicum, Pedaliaceae) seeds, or beech (Fagus sylvatica, Fagaceae) nuts date back to the $19^{\text {th }}$ century.

In 1981, the death of more than 600 people in Spain was attributed to the consumption of a product sold as olive oil. Investigations led to the belief that it was actually canola seed oil which had been denatured by the addition of aniline to make it suitable for industrial use. Fraudulent operators had tried to refine the oil and had sold it as olive oil.

In 2007, an article on the olive oil trade in The New Yorker magazine described a shipping tanker in 1991 in Ordu, Turkey loading several hundred tons of hazelnut oil for which the ship's official documents listed Greek olive oil. It was delivered to an Italian olive-oil producer and sold as olive oil.

A major study from the University of California, Davis in 2010 illustrates the degree of fraudulent olive oil sales in the United States, the world's largest importer of olive oil. From 14 imported brands and five Californian brands of EVOO, purchased from retail stores in three regions of California, a total of $69 \%$ of imported olive oil samples and $10 \%$ of California olive oil samples, labeled as EVOO, failed to meet the IOC and California Olive Oil Council sensory (organoleptic) standards for EVOO. Many of these also failed either IOC ultraviolet (UV) tests and/or pyropheophytin (PPP) and diacylglycerols (DAG) limits. The report was questioned by various importers in defense of their product, and the study was repeated with more stringent controls. In this study, five imported brands were sampled from each of 18 retail outlets. Of the five top-selling imported "extra virgin" olive oil brands in the United States, 66 of 90 of the samples analyzed again failed the IOC sensory standards for EVOOs analyzed by two IOC accredited sensory panels. Although EVOO deteriorates 
in store shelves if they are subject to light or have been exposed to high temperature, such as transportation in midsummer, it is expected that the oil will be EVOO quality when consumed.

There is an abundance of published reports on olive oil adulteration over the past decade, which is often covered by the mainstream media based on evidence provided after olive oil analysis and subsequent regulatory actions by government authorities. The Olive Oil Times, a digital news outlet covering the olive oil sector, has an entire section devoted to olive oil fraud. Most of these cases involve the sale of lower grade olive oil as EVOO or virgin olive oil, or misrepresenting the country of origin. Other articles have reported on the sale of olive oil mixed with lowercost vegetable oils, sometimes with the addition of chlorophyll, $\beta$-carotene, or other unidentified dyes.

In 2018, the Spanish company Dcoop was accused of blending canola and olive oil and selling it in the United States as Pompeian ${ }^{\circledR}$ brand. The label on Pompeian OlivExtra Original bottles described the oil as "composed of $85 \%$ canola oil and "First Cold Press Extra Virgin Oil". However, it is reported that the oil contained almost all Canadian canola oil with less than $1 \%$ being EVOO.

In April 2018, it was reported that Deoleo USA had paid seven million US dollars to settle a class action in California. Deoleo had allegedly mislabeled Bertolli ${ }^{\circledR}$ olive oil as "Imported from Italy" whereas Deoleo imported olive oil from several countries including Morocco, Tunisia, Greece, and Turkey. Additionally, the oil failed to meet standards limit for EVOO up to the "best by" date.

\section{Known adulterants:}

a. The most valuable grade of olive oil is EVOO. The most common adulterant found in EVOO are inferior grades of virgin or refined olive oil, as these grades are often products which have failed either the chemical or the sensory (organoleptic) standard to meet the standards of the higher-priced EVOO. They may be blended with EVOO and marketed fraudulently as EVOO. Virgin or refined olive oil have a poorer flavor and a much lower level of the antioxidants for which EVOO is renowned and for which it is purchased. Consumers may think they are getting the organoleptic and nutritional benefits of EVOO, but they are actually consuming an inferior product.

b. Other lower-cost virgin oil may also contain refined oil or pomace oil to obtain a greater financial return for the producer. In these cases, the adulterant may contain products from solvent extraction or refining that are not characteristic of olive oil, including traces of hexane solvent or trans fatty acids and glycidyl fatty acid esters. Trans fatty acids and fatty acid esters are generated at high temperature mainly during the deodorization process. Due to the carcinogenic property of glycidol, a tolerance value of $1 \mathrm{ppm}$ was recently established in Europe for food grade oils. c. Seed oils are significantly less expensive than olive oil and are commonly used as adulterants for EVOO or refined olive oil blends. The most common seed oils include canola/ rapeseed/colza, sunflower, and soybean oils. The mixture of seed oils and EVOO produce a product that has a different fatty acid profile to that of EVOO. It is also likely to have reduced antioxidants, as seed oils are generally refined.

d. Nut-based oils are also used to adulterate EVOO and refined olive oil blends. In particular, hazelnut oil has been reported to be used in blends with Italian olive oil. Hazelnut oil is a concern for authorities as it is difficult to detect levels of less than $10 \%$ in EVOO.

e. The green pigment, chlorophyll, is highest in newly extracted olive oil and gives the oil a fresh green appearance. This color is much lower or non-existent in refined or old oil. Chlorophyll can be chemically modified with copper to produce a pigment with a permanent green color and this can be added to old oil to give a perception of freshness and higher quality. Similarly, the orange pigment $\beta$-carotene has been found to be added to olive oil to impart a more desirable color. EVOO has a reasonably long shelf life when compared with other oils and will deteriorate over time as antioxidants are depleted and the peroxide value and free fatty acids increase. The useful life of EVOO can range from 12 to 24 months, depending on quality of the fruit and oil when it is pressed. This oil may be labeled with "use by" dates in excess of the oil life, or the oil may simply be sold as EVOO even though its quality has diminished. The sale of this oil is also fraudulent as it does not provide the health benefits and nutritive value expected of EVOO and declared on the label.

\section{Detection of adulteration of EVOO Analytical Methods and References for EVOO as Described by IOC}

A summary of analytical methods and references for EVOO as described by the International Olive Council [3] to determine the grade of olive oil, or possible adulteration, is shown in Table 1.

If a particular fault is perceived, the most relevant tests can be selected. Based on Table 1, those tests may reveal the following findings:

- Oil produced from poor quality fruit or poor processing conditions: If the fruit is damaged by disease, frost damage, insects or merely by harsh harvest and storage conditions, there will be an increase in free fatty acids and possibly increased peroxide value. In this case, tests $1,2,15$ and DAGs would be employed.

- Poor quality olive oil labeled as EVOO: Olive oil has to meet certain criteria to be labeled as EVOO. In particular, it must meet the sensory requirements of fruitiness and no defects. It must also be within prescribed limits for free fatty 
Tabel 1: Analytical Methods and References for EVOO as Described by the International Olive Council

\begin{tabular}{|c|c|}
\hline № & Title \\
\hline 1 & Determination of the free acidity according to COI/T.20/Doc. No 34/Rev.1, "Determination of free fatty acids, cold method" \\
\hline 2 & $\begin{array}{l}\text { Determination of the peroxide value according to COI/T.20/Doc. No 35/Rev.1 "Determination of the peroxide value", ISO 3960, or AOCS } \\
\text { Cd 8b-90 }\end{array}$ \\
\hline 3 & $\begin{array}{l}\text { Determination of the fatty acid composition and trans fatty acid content according to COI/T.20/Doc. No 33/Rev.1: "Determination of fatty } \\
\text { acid methyl esters by gas-chromatography" }\end{array}$ \\
\hline 4 & $\begin{array}{l}\text { Trans fatty acid content (\% trans fatty acids) C18:1 T C18:2 T + C18:3 T according to COI/T.20/Doc. No 33/Rev.1: "Determination of } \\
\text { fatty acid methyl esters by gas-chromatography" }\end{array}$ \\
\hline 5 & $\begin{array}{l}\text { Determination of the sterol content and alcoholic compounds according to COI/T.20/Doc. No 26/Rev.3: "Determination of the sterol } \\
\text { content and alcoholic compounds by capillary gas chromatography" }\end{array}$ \\
\hline 6 & $\begin{array}{l}\text { Erythrodiol and uvaol content (\% total sterols) according to COI/T.20/Doc. No. 30/Rev. 1: Determination of the composition and content } \\
\text { of sterols and triterpene diols by capillary column gas chromatography" }\end{array}$ \\
\hline 7 & Determination of wax content by capillary column, gas-liquid chromatography according to COI/T.20/DOC. 18 \\
\hline 8 & $\begin{array}{l}\text { Determination of the difference between the actual and theoretical ECN } 42 \text { triacylglycerol content according to COI/T.20/Doc. No 20/ } \\
\text { Rev.4, "Determination of the difference between actual and theoretical content of triacylglycerols with ECN } 42 \text { ", or AOCS 5b-89 }\end{array}$ \\
\hline 9 & $\begin{array}{l}\text { Determination of the stigmastadiene content according to COI/T.20/Doc. No 11/Rev.3, "Determination of stigmastadienes in vegetable } \\
\text { oils", or COI/T.20/Doc. no. 16/Rev.1, "Determination of sterenes in refined vegetable oils", or ISO 15788-1 or AOCS Cd 26-96 }\end{array}$ \\
\hline 10 & $\begin{array}{l}\text { Determination of the content of 2-glyceryl monopalmitate according to COI/T.20/Doc. No 23/Rev.1, "Determination of the percentage of } \\
\text { 2-glyceryl monopalmitate" or to ISO } 12872\end{array}$ \\
\hline 11 & $\begin{array}{l}\text { Determination of the unsaponifiable matter according to ISO 3596, "Determination of the unsaponifiable matter - Method using diethyl } \\
\text { ether extraction", or AOCS Ca 6b-53 or ISO } 18609 \text {. The results should be expressed in g unsaponifiable matter/kg oil }\end{array}$ \\
\hline 12 & $\begin{array}{l}\text { Determination of the content of waxes and alkyl esters according to COI/T.20/Doc. No 28/Rev.2, "Determination of the content of waxes, } \\
\text { fatty acid methyl esters and fatty acid ethyl esters by capillary gas chromatography" }\end{array}$ \\
\hline 13 & Determination of the organoleptic characteristics according to COI/T.20/Doc. No 15/Rev.10, "Organoleptic assessment of virgin olive oil" \\
\hline 14 & $\begin{array}{l}\text { Detection of trace metals according to ISO } 8294 \text {, "Determination of copper, iron and nickel by direct graphite furnace atomic absorption } \\
\text { spectrometry" }\end{array}$ \\
\hline 15 & $\begin{array}{l}\text { Determination of the absorbency in ultra-violet according to COI/T.20/Doc. No 19/Rev.4, "Spectrophotometric investigation in the ul- } \\
\text { traviolet", or ISO } 3656 \text { or AOCS Ch 5-91 }\end{array}$ \\
\hline
\end{tabular}

acids, peroxide value and UV absorbency. Therefore, tests 1, 2, 13, 15, as well as PPP and DAGs, would be applied.

- Refined olive oil labeled as virgin olive oil: Oil is refined generally to remove undesirable components such as free fatty acids and peroxides, which are the result of the oil's deterioration. Refining may also be used to remove natural pigments and/or products which may give the crude oil unpleasant flavor or odor, as in the case of crude rapeseed oil. Refining generally has three steps: refining, bleaching, and deodorizing (RBD). These steps, particularly deodorization, involve application of heat to the oil. When oil is heated, there are some common changes which can be detected by testing. Fatty acids, which make up the majority of the oil, can change their configuration from cis to trans fatty acids. Heating proportions of proportions of PPP and DAG in the oil. To determine the presence of refined oil, tests 4, 9, PPP, and DAG should be used.
- EVOO mixed with seed and nut oils: Seed oils are often refined and therefore tests applied to refined oil would also apply here. However, a more appropriate test would be to measure parameters specific for individual species. For example, the fatty acid composition and the sterol profile are generally good "fingerprints" for specific species. Canola oil, for example, has around $5-10 \%$ linolenic acid in the fatty acid profile whereas olive oil has less than $1 \%$ by IOC standards. Canola oil also contains a phytosterol named brassicasterol. Brassicasterol is not present in plant species other than those of the genus Brassica in the family Brassicaceae. A range of tests can be applied to determine the presence of seed or nut oils based on the fatty acid composition and sterols. These include tests $3,5,8,9,10,11$, and 15 .

- Solvent extracted oils including "pomace oil" and seed oils: Solvent extraction, generally with hexane or a similar solvent, is a much more efficient extraction process then merely squeezing or centrifuging the olive paste. However, by 
definition, solvent is not permitted in EVOO extraction, and it requires heating to remove any residual solvent traces from the oil. No solvents or extraneous chemicals are permitted in the processing of virgin olive oil. As solvents remove additional compounds from the olive fruit not extracted by regulation methods, analysis for the presence of these compounds can show if solvents have been used. Only traces of wax from the fruit skin are extracted by mechanical means but the wax will dissolve in the extraction solvent. Oils containing excessive amounts of wax are obtained by solvent extraction. The pentacyclic triterpenes erythrodiol and uvaol are extracted from olive pits (seeds), and the presence of these compounds also indicates solvent extraction. Tests to determine solvent extraction include 6 and 7.

- Old or badly stored olive oil sold as EVOO: Oil degrades over time, and the by-products are generally similar to that seen in oils that have been heated. Heating causes accelerated aging. Some of the tests used for refined oil can also be used to test for old, or badly stored, oil. Free fatty acids may increase slightly with time. Sensory characteristics will change with a decrease in fruitiness and an increase in defects as the oil becomes rancid. PPP and DAG proportions also change, and these tests are particularly useful to determine aging or poor storage conditions. Tests include 1, 2, 13,15, PPP, and DAG.

\section{Additional Analytical Methods and References for EVOO as Described by ISO}

Pyropheophytin and 1,2-diacylglycerol content:Two methods published by the International Organization for Standardization (ISO) involve the determination of the proportions of PPP and DAG in virgin olive oil. A recent study highlights the value of PPP and DAG indicators of olive oil quality and freshness. There is also a strong correlation with Organoleptic defects. The measurement of pyropheophytins helps detect deodorized olive oils and, together with DAG, is effective in determining oil storage conditions and aging.

International Standards: Each country may have its own definition of olive oil and its own limits for particular oil parameters and monitoring guidelines. Generally, these standards are based on the IOC and Codex standards [4] but often there are alternatives. Individual standards include the following:

- Codex Alimentarius: Standard for Olive Oils and Olive Pomace Oils. CXS 33-1981. Adopted in 1981. Revised in 1989, 2003, 2015. Amended in 2009, 2013 [5].

- European Union: Commission Regulation (EEC) No 2568/91 of 11 July 1991 on the characteristics of olive oil and olive- residue oil and on the relevant methods of analysis. Consolidated version: 04/12/2016 [6].

- International Olive Council: Trade Standard. COI/T.15/ NC No 3/Rev. 11. 2016 [7].
- United States: Standards for Grades of Olive Oil and Olive-Pomace Oil [8].

- State of California: Department of Food and Agriculture. Grade and Labeling Standards for Olive Oil, Refined-Olive Oil and Olive-Pomace Oil [9].

- Australian Standard: Olive oils and olive-pomace oils. AS 5264-2011 [10].

- Argentina: Código Alimentario Argentino. Capítulo VII. Alimentos Grasos. Aceites Alimenticios. Artículos 535 y 536 [11].

- South Africa: South African National Standard. Olive oils and olive-pomace oils. SANS 1377:2015 Edition 1 [12].

- Brazil: Ministério da Agricultura, Pecuária e Abastecimento. Gabinete do Ministro. Instrução normativa No 1, 30 de Janeiro de 2012 [13].

In a comparison of international standards, grades, limits, and tests for several countries were identified. In many cases, olive oil might be accepted in some countries as EVOO but rejected in others. This lack of uniformity creates trade barriers and is a further complication in fighting fraud.

\section{Detection of Adulteration of EVOO using FTIR Data Analysis}

Recent developments in Fourier transform infrared (FTIR) spectroscopy instrumentation extend the application of this technique to the field of food research, facilitating particularly the studies on edible oils and fats. In this work, FT-IR spectroscopy is used as an effective analytical tool in order to determine extra virgin olive oil adulteration with sunflower, corn and refined olive oils in their binary admixtures in different concentrations $(0,5,10,20,30,40,50$, $100 \%-\mathrm{w} / \mathrm{w})$. The spectral region $(1300-1000 \mathrm{~cm}-1)$ which contains the IR fingerprints of these vegetable oils was found to be very useful in detecting olive oil adulteration. A band shift observed at $3009 \mathrm{~cm}-1$ assigned to the $=\mathrm{C}-\mathrm{H}$ stretching vibration of the cis- double bond, allows the determination of extra virgin olive oil adulteration. The intensities of the spectral bands at $1163 \mathrm{~cm}-1$ (assigned to $-\mathrm{C}-\mathrm{O}$ stretching vibration and $\mathrm{CH} 2$ bending vibration) increase with increasing adulterant concentration. The absorbance ratio (R1118/1097 cm-1) decreased with increasing adulterant concentration. Also, there was a pronounced shift of the peak at $912.78 \mathrm{~cm}-1$ (assigned to $-\mathrm{HC}=\mathrm{CH}$ - cis- double bond, bending out of plane) for pure olive oil to higher wave numbers with increasing adulterant concentration [14].

Figures 1-4 display these spectral fingerprints in which one can visualize easily that the intensity of the absorption peak at $1163 \mathrm{~cm}-1$ decreases by increasing the concentration of sunflower, corn, and refined olive oils, respectively. 


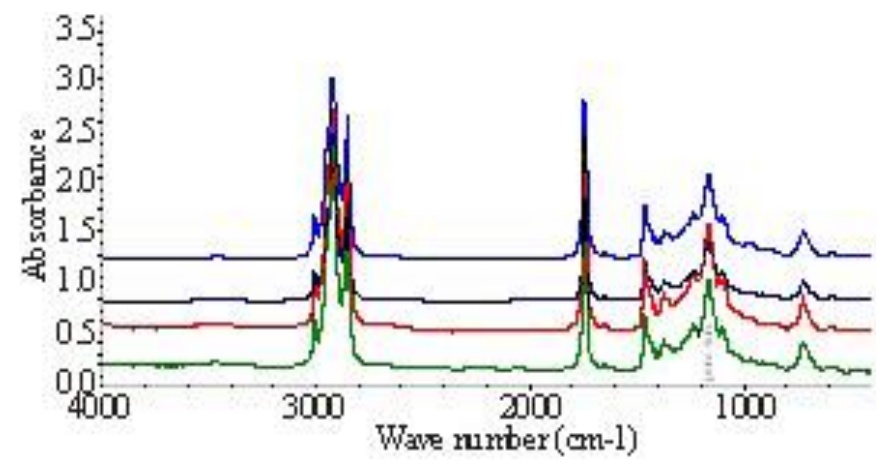

$100 \%$ sunflower oil $100 \%$ corn oil $100 \%$ extra virgin olive oil $100 \%$ refined olive oil

Figure 1: Typical FTIR Absorption spectra (4000-400 $\left.\mathrm{cm}^{-1}\right)$ of pure oils (sunflower, corn, extra virgin olive and refined olive oils)

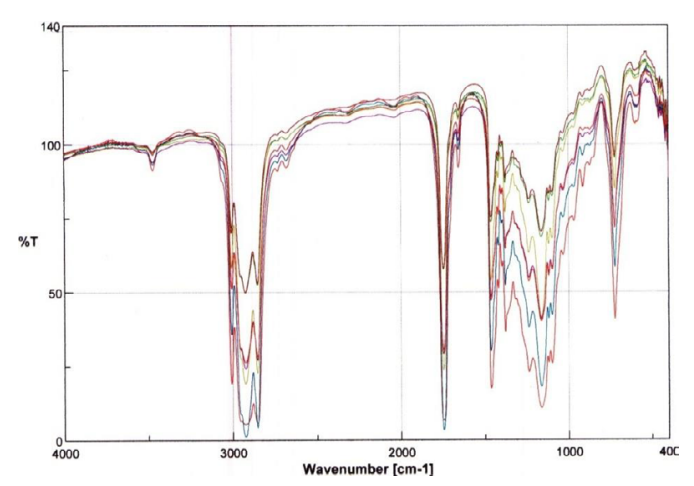

Figure 2: Typical FTIR spectra of pure olive oil (top) mixed with different ratios $(0,5,10,20,30,40,50$ and downwards till 100\%) of sunflower oil (bottom).

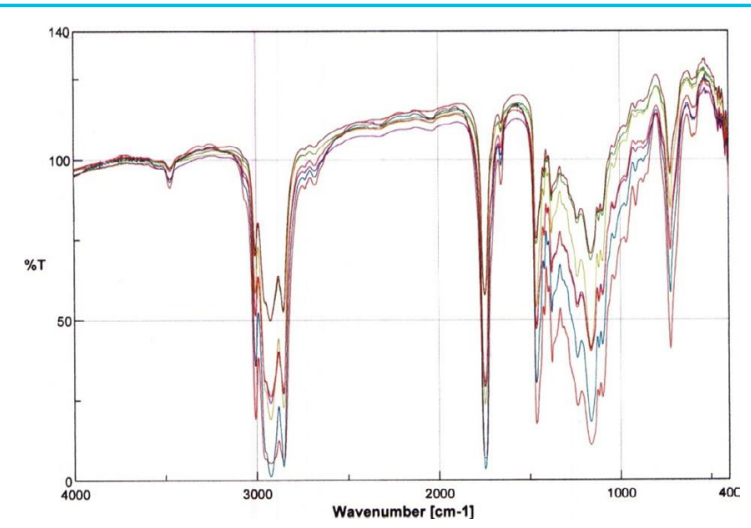

Figure 3: Typical FTIR spectra of pure olive oil (top) mixed with different ratios $(0,5,10,20,30,40,50$ and downwards till 100\%) of corn oil (bottom). 


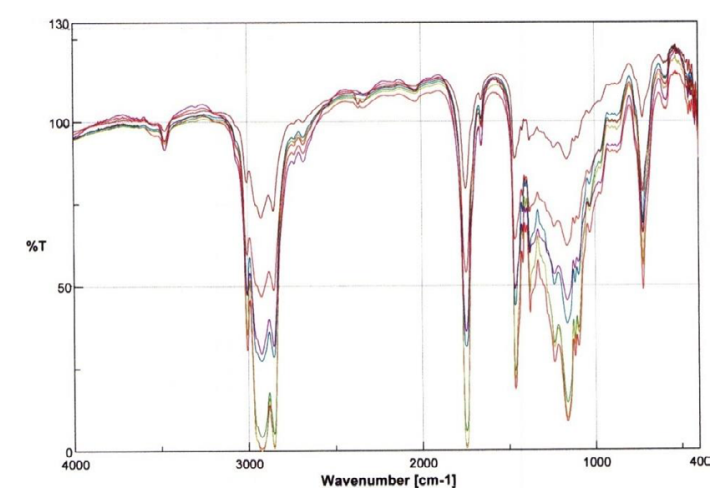

Figure 4: Typical FTIR spectra of pure olive oil (top) mixed with different ratios

$(0,5,10,20,30,40,50$ and downwards till 100\%) of refined olive oil (bottom).

\section{Detection of Adulteration of EVOO using UV Spectroscopy}

Spectroscopic examination in the ultraviolet (UV) can provide information on the quality of an oil, its state of preservation and changes brought about by technological processes. The absorption at the wavelengths $232 \mathrm{~nm}$ and 270 $\mathrm{nm}$ in cyclohexane is due to the presence of conjugated diene and triene systems, respectively, resulting from oxidation processes and/or refining practices. These absorptions are expressed as specific extinctions conventionally indicated by $\mathrm{K}$ (also referred to as "extinction coefficient"). In this work, the specific extinctions at $232 \mathrm{~nm}$ and $270 \mathrm{~nm}$ in cyclohexane are calculated for a concentration of $1 \% \mathrm{w} / \mathrm{v}$ for extra virgin olive oil adulteration with sunflower, corn oils in their binary admixtures in different concentrations $(0,5,15,25$ and $100 \%$ $\mathrm{w} / \mathrm{w})$. According to the regulations and recommendations stated in the official Commission Regulation (EEC) No $2568 / 91$ of 11 July 1991 on the characteristics of olive oil and olive- residue oil and on the relevant methods of analysis [6], the extinction coefficients K232 and K270 for extra virgin olive oil (EVOO) should be $\leq 2.5$ and $\leq 0.22$, respectively and $\Delta \mathrm{K}$ should be $\leq 0.01$. Results revealed that the adulteration will be detected easily by UV absorbance at $270 \mathrm{~nm}$ because sunflower oil showed a shift out of the permitted range at $15 \%$ upward higher concentrations added to extra virgin olive oil. Also, corn oil will be detected if present in $15 \%$ upward higher concentrations in blends with extra virgin olive oil. The calculated $\Delta \mathrm{K}$ allowed the detection of adulteration in extra virgin olive oil at the concentration of $15 \%$ upward higher concentrations of adulterant sunflower and corn oils; and this is the lowest detectable value in adulterated extra virgin olive oil [15].

\section{Detection of Adulteration of EVOO using Differential Scanning Calorimetry (DSC)}

The aim of this research was to identify the added refined olive oil (ROO) to extra virgin olive oil. One sample of each oil was analyzed, admixtures of EVOO: ROO were prepared at different ratios (50:50,60:40, 70:30, 80:20, 90:10 and $95: 5 \mathrm{w} / \mathrm{w}$ ). To identify this adulteration, exothermic and endothermic thermograms were investigated. The results indicated that by increasing refined olive oil, onset temperature (Ton) was increased from -61.08 to $-56.16{ }^{\circ} \mathrm{C}$; also, offset temperature (Toff) decreased from 19.09 to 12.57 ${ }^{\circ} \mathrm{C}$. Also results showed that when the ROO ratio increased then the enthalpy average values of both exothermic and endothermic curves were declined. This matter is subject to changes in fatty acids, since by adding more refined olive oil, the amount of oleic acid, which is a monounsaturated fatty acid was increased, and the amount of linoleic acid, which is polyunsaturated fatty acid, decreased. Ton relates to melting monounsaturated triacylglycerols and Toff relates to crystallization of polyunsaturated triacylglycerols [16].

\section{Detection of Adulteration of EVOO using UHPLC- CAD profiling of triacylglycerols and PCA}

Ultra-high-performance liquid chromatography (UHPLC) with charged aerosol detection (CAD) was employed to characterize EVOO along with potential adulterant oils based on their triacylglycerol (TAG) profiles. Statistical analysis of these TAGs using principal component analysis (PCA) allows for a rapid approach to determine EVOO authenticity. Using this approach, adulteration of EVOO with cheaper vegetable and seed oils and lower-quality olive oils had detection limits at or below $10 \%$, depending on the adulterant. Compared to traditional methods, UHPLC-CAD with PCA involves minimal sample preparation combined with fast analysis, for a rapid determination of EVOO authenticity [17].

\section{CONCLUSIONS}

The adulteration of olive oil, particularly EVOO, is prolific because the financial gains are large and the availability of the highest grades low. Seed oils from broad-acre farming are relatively inexpensive due to the lower cost of production and the more efficient (solvent extraction) methods of processing. Lower quality olive oils are more abundant and cheaper to produce. There is a significant financial gain for fraudsters who might blend seed oils or refined/lower quality olive oils and sell them as EVOO. Because testing is sporadic and expensive, the chance of being caught is relatively remote and 
the potential consequences not severe under the law of most countries. Blending a small portion of aromatic EVOO with tasteless, colorless, refined seed oil can produce a product with some sensory and visually characteristics that make it appear to be authentic. Adulteration of EVOO with seed oil and/or refined and/or poor quality olive oil is not known to cause severe health issues (with the exception of the toxic oil syndrome cases in 1981). However, the main issue is more likely the decline in health benefits due to degradation of beneficial components of EVOO. Despite this, consumers continue to buy olive oil for the perceived and documented nutritional benefits and for the sensory qualities of freshness and fruitiness. Clearly, a more determined global effort from authorities is required to overcome the corrupt practices. More testing of products, particularly from government facilities, is warranted to ensure the oils meet the expectations expressed on the label. There needs to be more government support in export/import situations and higher penalties for those that abuse the regulations.

\section{References}

1. Mailer, R.J., Gafner, S. (2020). Adulteration of olive oil. Botanical Adulterants Prevention Bulletin.Austin, TX: ABC-AHP-NCNPR Botanical Adulterants Prevention Program; 2020.

2. Olive Oil Times (2020). https://www.oliveoiltimes.com/production/oliveoil-consumption-outpace-production/86856

3. Testing methods (2019). http://www.internationaloliveoil.org/estaticos/ view/224-testing-methods. Accessed April 24, 2019.

4. Mailer, R.J. (2014). A world tour of olive oil standards. 105th AOCS Annual Meeting and Short Course; 2014; San Antonio, TX.

5. Joint WHO/FAO Codex Alimentarius Commission (2015). Codex Alimentarius: Standard for olive oils and olive pomace oils. Vol CODEX STAN 33-1981. Rome, Italy: World Health Organization and Food and Agriculture Organization of the United Nations; 2015:1-9.
6. Commission of the European Communities (2016). Commission Regulation (EEC) No 2568/91 of 11 July 1991 on the characteristics of olive oil and olive-residue oil and on the relevant methods of analysis. Brussels, Belgium: European Economic Community; 1991:1-128.

7. International Olive Council (2018). Trade standard applying to olive oils and olive pomace oils. Vol COI/T.15/NC No 3/Rev. 12. Madrid: International Olive Council; 2018:17.

8. United States standards for grades of olive oil and olive-pomace oil (2010). Washington, DC: United States Department of Agriculture; 2010:1-19.

9. Grade and labeling standards for olive oil, refined-olive oil and olivepomace oil (2014). Sacramento, CA: California Department of Food and Agriculture; 2014:1-14.

10. Committee FT-034 Olive Oils (2011). Australian Standards: olive oil and olive pomace oils. Sidney, NSW, Australia: Standards Australia; 2011:1-7.

11. Código Alimentario Argentino (2018). Capítulo VII. Alimentos Grasos, Aceites Alimenticios. Buenos Aires, Argentina: Ministerio de Salud y Desarrollo Social; 2018.

12. South African National Standard: Olive oils and olive-pomace oils (2015). SANS 1377:2015. Pretoria, South Africa: SABS Standards Division 2015.

13. Instrução normativa No 1, 30 de Janeiro de (2012). Brasilia, Brazil: Ministério da Agricultura, Pecuária e Abastecimento; 2012.

14. Almoselhy R.I.M., Allam M.H., Kotb, A.A., El-Sharkawy, A. A. (2009). Detection of Adulteration of Extra Virgin Olive Oil using FTIR Data Analysis. Research Bulletin, Ain Shams Univ., № 13, 2009 - 12 pages.

15. Hashem, H., Almoselhy, R.I.M., El-Waseif, M., Magdy, A. (2020). Rapid Authentication of Extra Virgin Olive Oil using UV and FTIR Spectroscopy. Middle East Journal of Applied Sciences, 10 (2): 263-271.

16. Karbasian, M., Givianrad, M.H., Ramezan, Y. (2015). A Rapid Method for Detection of Refined Olive Oil as Adulterant in Extra Virgin Olive Oil by Differential Scanning Calorimetry. Orient. J. Chem., Vol. 31(3), 1735-1739.

17. Green, H.S., Li, X., Pra, M.D., Lovejoy, K.S., Steiner, F., Acworth, I.N., Wang, S.C. (2020). A rapid method for the detection of extra virgin olive oil adulteration using UHPLC-CAD profiling of triacylglycerols and PCA, Food Control, 107, 106773. 